\title{
Effects of repeated restraint and blood sampling with needle injection on blood cardiac troponins in rats, dogs, and cynomolgus monkeys
}

\author{
Keisuke Nagata $^{1} \cdot$ Kazutoshi Sawada $^{2} \cdot$ Hirofumi Minomo ${ }^{2}$ Daisuke Sasaki ${ }^{1}$. \\ Katsuyuki Kazusa $^{1} \cdot$ Kazuhiko Takamatsu $^{1}$ (D)
}

Received: 3 April 2017 / Accepted: 16 August 2017 / Published online: 24 August 2017

(C) The Author(s) 2017. This article is an open access publication

\begin{abstract}
While cardiac troponins (cTnT and cTnI) have been used as blood biomarkers of myocardial injury such as myocardial infarction in both humans and animals, their high diagnostic sensitivity inevitably leads to decreased diagnostic specificity. For example, it is difficult to judge whether a slight increase of cardiac troponins in toxicological studies is a treatment-related response or not. Drawing an accurate conclusion requires reliable background data and definitive criteria based on that data. However, no organized efforts in setting such criteria has been reported. Here, we measured blood cTnI and cTnT concentrations in Sprague-Dawley rats, beagle dogs, and cynomolgus monkeys from repeated blood samplings using needle cylinders under restraint up until $24 \mathrm{~h}$ after a single oral dose of $0.5 w / v \%$ methyl cellulose solution as a vehicle. We revealed the extent of individual differences in baseline levels and operational effects. Our results can be useful in making criteria for judgment of treatment-related changes in cardiac troponins.
\end{abstract}

Keywords Cardiac troponin $\cdot \mathrm{cTnT} \cdot \mathrm{cTnI} \cdot$ Myocardial injury $\cdot$ Biomarker

Kazuhiko Takamatsu

kazuhiko.takamatsu@astellas.com

1 Drug Safety Research Laboratories, Astellas Pharma Inc., 21 Miyukigaoka, Tsukuba, Ibaraki 305-8585, Japan

2 Drug Safety Research Laboratories, Shin Nippon Biomedical Laboratories, Ltd., 2438 Miyanoura, Kagoshima 891-1394, Japan

\section{Introduction}

Cardiac troponins (cTnT and cTnI) are used as clinical blood biomarkers for myocardial injuries such as myocardial infarction (Mahajan and Jarolim 2011) since they have high diagnostic sensitivity and tissue specificity. Since their structure and function are highly conserved across species (O'Brien et al. 2006), cardiac troponins are also used as translational biomarkers in experimental studies in animals (Berridge et al. 2009; Hausner et al. 2013; Herman et al. 2001; Newby et al. 2011; Pierson et al. 2013; Undhad et al. 2012). However, despite the usability of troponins in cardiac injuries, their high diagnostic sensitivity still poses a challenge since increased diagnostic sensitivity inevitably results in decreased diagnostic specificity (i.e., an increased number of false positives) (Mahajan and Jarolim 2011). In particular, when they are applied in toxicological studies, it is often difficult to distinguish treatment-related changes from operational changes. Therefore, obtaining data about blood cardiac troponin levels in intact animals is extremely important.

Schultze et al. previously reported blood $\mathrm{cTnI}$ measurements in intact Sprague-Dawley rats and cynomolgus monkeys. Their experiments consisted of careful measurements made over multiple time points under resting conditions after saline administration by oral gavage (Schultze et al. 2009, 2015). Although these studies provided much-needed data for future cTnI research, serial blood samplings were conducted using an automated cannulation method, which is different from the standard procedures of most toxicity studies (i.e., repeated needle injections under restraint).

Here, we aimed to obtain background data in a setting similar to that of typical pharmaceutical toxicological studies conducted in animals. We measured blood cTnI and cTnT concentrations in Sprague-Dawley rats, beagle dogs, and cynomolgus monkeys, from repeated blood samplings 
using needle cylinders under restraint up until $24 \mathrm{~h}$ after a single oral dose of $0.5 \mathrm{w} / \mathrm{v} \%$ methyl cellulose solution as vehicle. In addition, for dogs and cynomolgus monkeys, we also measured creatine kinase (CK) and lactate dehydrogenase (LDH) to monitor the extent of struggle during the restraint.

\section{Material and methods}

\section{Animals experiments}

Rats Seven-week-old male and female Sprague-Dawley rats (Crl:CD (SD)) supplied from Charles River Japan Inc. (Tokyo, Japan) were used. Animals were kept in bracket-type stainless steel wire-meshed cages (two or three animals per cage during the study period) at a temperature of $23 \pm 3{ }^{\circ} \mathrm{C}$ and relative humidity of $55 \pm 15 \%$ with illumination of $12 \mathrm{~h}$ per day from 7 a.m. to 7 p.m. Animals could freely access CRF-1 pellet diet (Oriental Yeast Co., Ltd. (Tokyo, Japan)) and drinking water. Animals were quarantined and acclimated for 2 weeks. Five male and female animals were treated with a single oral dose of $0.5 \mathrm{w} / \mathrm{v} \%$ methyl cellulose solution $(5 \mathrm{~mL} / \mathrm{kg})$ using flexible stomach tubes and syringes. Around $0.25 \mathrm{~mL} /$ animal of blood was collected via tail vein while conscious and restrained at $0.5,1,2,4$, and $8 \mathrm{~h}$ after the treatment. In addition, around $2 \mathrm{~mL} /$ animal of blood was collected via the abdominal aorta under anesthesia with isoflurane $24 \mathrm{~h}$ after the treatment. Blood samples collected in sodium heparin tubes were immediately placed on ice, centrifuged by $10,000 \mathrm{rpm}$ at $4{ }^{\circ} \mathrm{C}$ for $2 \mathrm{~min}$ to obtain plasma, and stored at $-80{ }^{\circ} \mathrm{C}$ until measurement.

Dogs Ten- to 13-month-old male and female beagle dogs that had been supplied from Hongo Farm, Kitayama Labes Co., Ltd. (Yamaguchi, Japan) were used. Animals were kept in stainless cages (one animal per cage) under the temperature of $23 \pm 3{ }^{\circ} \mathrm{C}$ and relative humidity of $50 \pm 20 \%$ with illumination of $12 \mathrm{~h}$ per day from 7 a.m. to 7 p.m. Animals were supplied with around $300 \mathrm{~g} /$ day of NVE-10 pellet diet (Nihon Pet Food (Tokyo, Japan)) and could freely access to and drinking water. Animals were acclimated to the test condition for 2 weeks, during which the animals were treated with drinking water $(30 \mathrm{~mL} /$ animal $)$ in the same manner as methyl cellulose solution. After that, 30 male and 30 female animals were treated with a single oral dose of $0.5 \mathrm{w} / \mathrm{v} \%$ methyl cellulose solution $(5 \mathrm{~mL} / \mathrm{kg})$ using disposable catheter and syringe. Around 7.8 (only at - D6) or $2.3 \mathrm{~mL} /$ animal per timepoint ( $0.3 \mathrm{~mL}$ for cTnT and $2 \mathrm{~mL}$ for the other items) of blood was collected via external jugular vein from conscious animals 6 days before the treatment ( - D6) and just before (Pre) and $0.5,1,2,4,8$, and $24 \mathrm{~h}$ after the treatment (D0). For the $\mathrm{cTnT}$ measurement, blood samples collected in sodium heparin tubes were immediately placed on ice until measurement. For the measurements of the other parameters, collected blood samples were placed at room temperature for 20-60 min, centrifuged (room temperature, $3000 \mathrm{rpm}$ for $10 \mathrm{~min}$ ) to obtain serum, and either measured within the same day or stored at $-70{ }^{\circ} \mathrm{C}$ until measurement.

Cynomolgus monkeys Three- to seven-year-old male and female cynomolgus monkeys that had been supplied from Angkor Primates Center Inc. (Kampong Thom, Cambodia) or Tian Hu Cambodia Animal Breeding Research Center Ltd. (Kampong Thom, Cambodia) were used. Animals were kept in stainless cages (one animal per cage) at a temperature of $26 \pm 3{ }^{\circ} \mathrm{C}$ and relative humidity of $50 \pm 20 \%$ with illumination of $12 \mathrm{~h}$ per day from 7 a.m. to 7 p.m. Animals were supplied with around $108 \mathrm{~g} /$ animal/day of HF Primate J 12G pellet diet (Purina Mills, LLC. (MO, USA)) and could freely access to drinking water. Animals were acclimated to the test conditions for 2 weeks, during which the animals were treated with drinking water $(10 \mathrm{~mL} / \mathrm{animal})$ in the same manner as methyl cellulose solution. After that, 10 male and 10 female animals were treated with a single oral dose of $0.5 \mathrm{w} / \mathrm{v} \%$ methyl cellulose solution $(5 \mathrm{~mL} / \mathrm{kg})$ using disposable catheters and syringes. Around 4.5 (only at - D13) or $2.3 \mathrm{~mL} /$ animal per time point $(0.3 \mathrm{~mL}$ for $\mathrm{cTnT}$ and $2 \mathrm{~mL}$ for the other items) of blood was collected via femoral vein under unanesthetized condition and restraint in a restraint device 13 days before the treatment (-D13) and just before (Pre) and $0.5,1,2,4,8$, and $24 \mathrm{~h}$ after the treatment (D0). For the cTnT measurement, blood samples collected in sodium heparin tubes were immediately placed on ice until measurement. For the parameters of the other items, collected blood samples were placed at room temperature for 20-60 min, centrifuged (room temperature, $3000 \mathrm{rpm}$ for $10 \mathrm{~min}$ ) to obtain serum, and either measured within the same day or stored at $-80^{\circ} \mathrm{C}$ until measurement.

\section{Dosing formulation}

The requisite amount of methyl cellulose (Metlose ${ }^{\circledR}$ SM-400, Shin-Etsu Chemical Co., Ltd., Tokyo, Japan) was dissolved in water for injection (Otsuka Pharmaceutical Factory, Inc., Tokushima, Japan.) to make a concentration of $0.5 \mathrm{w} / \mathrm{v} \%$.

\section{Clinical testing methods}

cTnI and cTnT levels were measured in rats, dogs and cynomolgus monkeys. CK and LDH levels were also measured in dogs and monkeys to monitor the effect by strenuous movement. The measurement methods are as follows.

cTnI: For rats, plasma samples were measured with Cardiac Injury Panel 3 (rat) Assay Kit and SECTOR® 
Imager 6000 (Meso Scale Discovery, MD, USA). For dogs and cynomolgus monkeys, serum samples were measured with Multiskan Ascent (Thermo Fischer Scientific, MA, USA).

cTnT: For rats, plasma samples were measured with Cardiac Injury Panel 3 (rat) Assay Kit and SECTOR® Imager 6000 (Meso Scale Discovery, MD, USA). For dogs and cynomolgus monkeys, blood samples were measured with Cobas h 232 (Roche Diagnostics GmbH, Mannheim, Germany).

CK and LDH: Serum samples were measured with JCABM6070 (Nihon Denshi, Tokyo, Japan) in both dogs and cynomolgus monkeys.

Note that all the testing methods were validated for their intra-assay precision, inter-assay precision, and frozen stability.

\section{Results}

None of the study animals exhibited an abnormal general condition.

\section{Rats}

Plasma cTnI levels were below the lower limit of quantification (BLOQ) at almost all time points except for in one male (RM05) and two females (RF01 and RF02) $2 \mathrm{~h}$ after dosing, and one male (RM05) $4 \mathrm{~h}$ after dosing (Table 1). The detected levels were from 0.015 to $0.028 \mathrm{ng} / \mathrm{mL}$. All time points for plasma cTnT levels were BLOQ (Table 1).

\section{Dogs}

Serum cTnI levels were detected in almost all animals except for in 2 males (DM22 and DM27) (Table 2). Although the levels detected varied among individuals, a tendency for levels to be constant throughout the examination period was noted in animals that showed higher levels (DM12). For blood cTnT levels, one male (DM26) and five females (DF03, DF13, DF22, DF28, and DF29) showed detectable but lower levels throughout the examination period (Table 2). The other animals showed BLOQ at all points.

No animals showed abnormal LDH values throughout the examination period (Table 2). One male (DM13 and DM23) and two females (DF15 and DF16) showed higher CK values $8 \mathrm{~h}$ after dosing than those at pre-dosing (Table 2). No corresponding change to higher $\mathrm{CK}$ values were noted in $\mathrm{cTnI}$ or cTnT in these animals.
Table 1 Measurements in rats

\begin{tabular}{lllllll}
\hline \multicolumn{7}{c}{ cTnI measurements in male rats } \\
\multicolumn{7}{c}{ cTnI $(\mathrm{ng} / \mathrm{mL})$} \\
0D & & & & & \\
No. & $0.5 \mathrm{~h}$ & $1 \mathrm{~h}$ & $2 \mathrm{~h}$ & $4 \mathrm{~h}$ & $8 \mathrm{~h}$ & $24 \mathrm{~h}$ \\
$\mathrm{RM} 01$ & - & - & - & - & - & - \\
$\mathrm{RM} 02$ & - & - & - & - & - & - \\
RM03 & - & - & - & - & - & - \\
RM04 & - & - & - & - & - & - \\
RM05 & - & - & 0.028 & 0.015 & - & - \\
Mean & 0.000 & 0.000 & 0.006 & 0.003 & 0.000 & 0.000 \\
SD & 0.000 & 0.000 & 0.011 & 0.006 & 0.000 & 0.000
\end{tabular}

cTnT measurements in male rats $\mathrm{cTnT}(\mathrm{ng} / \mathrm{mL})$

$$
0 \mathrm{D}
$$

$\begin{array}{lllllll}\text { No. } & 0.5 \mathrm{~h} & 1 \mathrm{~h} & 2 \mathrm{~h} & 4 \mathrm{~h} & 8 \mathrm{~h} & 24 \mathrm{~h} \\ \text { RM01 } & - & - & - & - & - & - \\ \text { RM02 } & - & - & - & - & - & - \\ \text { RM03 } & - & - & - & - & - & - \\ \text { RM04 } & - & - & - & - & - & - \\ \text { RM05 } & - & - & - & - & - & - \\ \text { Mean } & 0.000 & 0.000 & 0.000 & 0.000 & 0.000 & 0.000 \\ \text { SD } & 0.000 & 0.000 & 0.000 & 0.000 & 0.000 & 0.000\end{array}$

cTnI measurements in female rats $\mathrm{cTnI}(\mathrm{ng} / \mathrm{mL})$

$$
0 \mathrm{D}
$$

$\begin{array}{lllllll}\text { No. } & 0.5 \mathrm{~h} & 1 \mathrm{~h} & 2 \mathrm{~h} & 4 \mathrm{~h} & 8 \mathrm{~h} & 24 \mathrm{~h} \\ \text { RF01 } & - & - & 0.021 & - & - & - \\ \text { RF02 } & - & - & 0.016 & - & - & - \\ \text { RF03 } & - & - & - & - & - & - \\ \text { RF04 } & - & - & - & - & - & - \\ \text { RF05 } & - & - & - & - & - & - \\ \text { Mean } & 0.000 & 0.000 & 0.007 & 0.000 & 0.000 & 0.000 \\ \text { SD } & 0.000 & 0.000 & 0.009 & 0.000 & 0.000 & 0.000\end{array}$

$\mathrm{cTnT}$ measurements in female rats $\mathrm{cTnT}(\mathrm{ng} / \mathrm{mL})$ $0 \mathrm{D}$

\begin{tabular}{lllllll} 
No. & $0.5 \mathrm{~h}$ & $1 \mathrm{~h}$ & $2 \mathrm{~h}$ & $4 \mathrm{~h}$ & $8 \mathrm{~h}$ & $24 \mathrm{~h}$ \\
RF01 & - & - & - & - & - & - \\
RF02 & - & - & - & - & - & - \\
RF03 & - & - & - & - & - & - \\
RF04 & - & - & - & - & - & - \\
RF05 & - & - & - & - & - & - \\
Mean & 0.000 & 0.000 & 0.000 & 0.000 & 0.000 & 0.000 \\
SD & 0.000 & 0.000 & 0.000 & 0.000 & 0.000 & 0.000 \\
\hline
\end{tabular}

cTnI: Values below the lower limit of quantification (BLOQ) $(0.010 \mathrm{ng} / \mathrm{mL})$ were shown as "-" and regarded as $0 \mathrm{ng} / \mathrm{mL}$ in calculation

cTnT: Values below the lower limit of quantification (BLOQ) (0.392 to $0.412 \mathrm{ng} / \mathrm{mL}$ ) were shown as "-_" and regarded as $0 \mathrm{ng} / \mathrm{mL}$ in calculation 
Table 2 Measurements in dogs

cTnI measurements in male beagles cTnI (ng/mL)

$\begin{array}{lllllllll} & -6 \mathrm{D} & 0 \mathrm{D} & & & & & & \\ \text { No. } & - & \text { Pre } & 0.5 \mathrm{~h} & 1 \mathrm{~h} & 2 \mathrm{~h} & 4 \mathrm{~h} & 8 \mathrm{~h} & 24 \mathrm{~h} \\ \text { DM01 } & - & - & 0.19 & - & - & 0.18 & - & - \\ \text { DM02 } & - & - & 0.19 & - & - & - & - & - \\ \text { DM03 } & - & - & - & - & 0.17 & - & 0.18 & - \\ \text { DM04 } & 0.16 & - & - & 0.18 & - & - & - & - \\ \text { DM05 } & - & - & - & - & - & - & 0.40 & - \\ \text { DM06 } & - & - & 0.38 & - & - & 0.20 & 0.31 & 0.25 \\ \text { DM07 } & - & - & 0.19 & 0.26 & 0.24 & 0.16 & - & 0.26 \\ \text { DM08 } & 0.73 & 0.71 & 0.55 & 0.78 & 0.41 & 0.85 & 1.17 & 0.58 \\ \text { DM09 } & 0.20 & - & - & - & 0.17 & - & 0.25 & 0.18 \\ \text { DM10 } & 0.35 & 0.33 & - & 0.32 & 0.38 & 0.43 & 0.23 & - \\ \text { DM11 } & - & 0.51 & 0.29 & 0.53 & 0.44 & 0.20 & - & 0.50 \\ \text { DM12 } & 3.69 & 3.04 & 3.16 & 3.80 & 3.83 & 3.79 & 3.88 & 3.63 \\ \text { DM13 } & 0.81 & 0.44 & 0.44 & 0.81 & 0.71 & 1.04 & 0.87 & 0.67 \\ \text { DM14 } & 0.36 & 0.37 & 0.31 & - & 0.58 & 0.37 & 0.78 & 0.42 \\ \text { DM15 } & - & 0.35 & - & - & - & 0.27 & 0.25 & 0.40 \\ \text { DM16 } & - & - & 0.45 & 0.41 & - & - & 0.50 & 0.33 \\ \text { DM17 } & - & 0.32 & - & 0.38 & 0.39 & - & - & 0.32 \\ \text { DM18 } & 0.52 & 0.37 & 0.40 & - & 0.71 & 0.26 & 0.36 & - \\ \text { DM19 } & 0.51 & 0.63 & 0.57 & 0.47 & 0.39 & 0.90 & 0.73 & 0.57 \\ \text { DM20 } & 0.57 & 0.44 & 0.57 & 0.44 & 0.35 & - & 0.95 & 0.38 \\ \text { DM21 } & - & 0.22 & - & 0.19 & - & - & - & - \\ \text { DM22 } & - & - & - & - & - & - & - & - \\ \text { DM23 } & - & - & - & - & - & 0.21 & - & - \\ \text { DM24 } & - & - & - & - & - & - & 0.57 & - \\ \text { DM25 } & - & 0.28 & 0.27 & 0.29 & 0.30 & 0.31 & 0.42 & 0.30 \\ \text { DM26 } & - & 0.56 & 0.57 & 0.70 & 0.69 & 0.70 & 0.72 & 0.66 \\ \text { DM27 } & - & - & - & - & - & - & 0.18 & - \\ \text { DM28 } & 0.31 & 0.31 & 0.20 & - & 0.35 & 0.25 & 0.35 & - \\ \text { DM29 } & - & 0.19 & - & - & - & - & - & - \\ \text { DM30 } & - & - & 0.31 & - & - & - & 0.39 & - \\ \text { Mean } & 0.27 & - & - & - & - & 0.29 & 0.17 & 0.38 \\ \text { SD } & 0.68 & - & - & - & & & & \end{array}$

cTnT measurements in male beagles

$$
\text { cTnT (ng/L) }
$$

\begin{tabular}{lllllllll}
\multicolumn{1}{r}{} & $-6 \mathrm{D}$ & $\mathrm{0D}$ & & & & \\
No. & - & Pre & $0.5 \mathrm{~h}$ & $1 \mathrm{~h}$ & $2 \mathrm{~h}$ & $4 \mathrm{~h}$ & $8 \mathrm{~h}$ & $24 \mathrm{~h}$ \\
DM01 & - & - & - & - & - & - & - & - \\
DM02 & - & - & - & - & - & - & - & - \\
DM03 & - & - & - & - & - & - & - & - \\
DM04 & - & - & - & - & - & - & - & - \\
DM05 & - & - & - & - & - & - & - & - \\
DM06 & - & - & - & - & - & - & - & - \\
DM07 & - & - & - & - & - & - & - & - \\
DM08 & - & - & - & - & - & - & - & - \\
DM09 & - & - & - & - & - & - & - & - \\
DM10 & - & - & - & - & - & - & - & -
\end{tabular}

Table 2 (continued)

\begin{tabular}{|c|c|c|c|c|c|c|c|}
\hline DM11 & - & - & - & - & - & - & - \\
\hline DM12 & - & - & - & - & - & - & - \\
\hline DM13 & - & - & - & - & - & - & - \\
\hline DM14 & - & - & - & - & - & - & - \\
\hline DM15 & - & - & - & - & - & - & - \\
\hline DM16 & - & - & - & - & - & - & - \\
\hline DM17 & - & - & - & - & - & - & - \\
\hline DM18 & - & - & - & - & - & - & - \\
\hline DM19 & - & - & - & - & - & - & - \\
\hline DM20 & - & - & - & - & - & - & - \\
\hline DM21 & - & - & - & - & - & - & - \\
\hline DM22 & - & - & - & - & - & - & - \\
\hline DM23 & - & - & - & - & - & - & - \\
\hline DM24 & - & - & - & - & - & - & - \\
\hline DM25 & - & - & - & - & - & - & - \\
\hline DM26 & - & + & 101 & + & + & + & + \\
\hline DM27 & - & - & - & - & - & - & - \\
\hline DM28 & - & - & - & - & - & - & - \\
\hline DM29 & - & - & - & - & - & - & - \\
\hline DM30 & - & - & - & - & - & - & - \\
\hline
\end{tabular}

CK measurements in male beagles CK (IU/L)

$\begin{array}{lllllllll} & -6 \mathrm{D} & 0 \mathrm{D} & & & & & & \\ \text { No. } & - & \text { Pre } & 0.5 \mathrm{~h} & 1 \mathrm{~h} & 2 \mathrm{~h} & 4 \mathrm{~h} & 8 \mathrm{~h} & 24 \mathrm{~h} \\ \text { DM01 } & 134 & 104 & 229 & 97 & 119 & 121 & 122 & 111 \\ \text { DM02 } & 100 & 104 & 161 & 94 & 108 & 139 & 132 & 88 \\ \text { DM03 } & 106 & 107 & 116 & 107 & 133 & 110 & 114 & 101 \\ \text { DM04 } & 102 & 97 & 82 & 74 & 159 & 108 & 186 & 106 \\ \text { DM05 } & 95 & 96 & 152 & 97 & 127 & 128 & 140 & 101 \\ \text { DM06 } & 107 & 96 & 300 & 120 & 86 & 93 & 97 & 96 \\ \text { DM07 } & 105 & 107 & 105 & 97 & 112 & 113 & 115 & 101 \\ \text { DM08 } & 104 & 125 & 198 & 148 & 105 & 109 & 184 & 178 \\ \text { DM09 } & 90 & 91 & 90 & 87 & 92 & 111 & 98 & 91 \\ \text { DM10 } & 97 & 110 & 122 & 127 & 136 & 150 & 146 & 135 \\ \text { DM11 } & 134 & 135 & 184 & 145 & 182 & 163 & 149 & 133 \\ \text { DM12 } & 1150 & 104 & 90 & 130 & 78 & 106 & 130 & 86 \\ \text { DM13 } & 145 & 102 & 106 & 108 & 129 & 198 & 330 & 113 \\ \text { DM14 } & 108 & 111 & 164 & 108 & 110 & 109 & 105 & 101 \\ \text { DM15 } & 112 & 95 & 100 & 102 & 104 & 103 & 110 & 73 \\ \text { DM16 } & 85 & 451 & 467 & 406 & 352 & 276 & 261 & 75 \\ \text { DM17 } & 103 & 93 & 102 & 125 & 102 & 108 & 150 & 83 \\ \text { DM18 } & 106 & 77 & 94 & 101 & 91 & 97 & 104 & 80 \\ \text { DM19 } & 104 & 110 & 145 & 149 & 164 & 176 & 273 & 114 \\ \text { DM20 } & 83 & 83 & 92 & 107 & 92 & 98 & 112 & 83 \\ \text { DM21 } & 128 & 164 & 178 & 159 & 187 & 219 & 217 & 122 \\ \text { DM22 } & 87 & 203 & 185 & 171 & 208 & 220 & 217 & 94 \\ \text { DM23 } & 99 & 123 & 133 & 132 & 123 & 144 & 344 & 90 \\ \text { DM24 } & 152 & 99 & 103 & 116 & 90 & 107 & 109 & 84 \\ \text { DM25 } & 96 & 102 & 89 & 93 & 96 & 104 & 86 & 80 \\ \text { DM26 } & 80 & 76 & 141 & 80 & 82 & 99 & 102 & 77 \\ \text { DM27 } & 133 & 141 & 152 & 152 & 160 & 171 & 152 & 116 \\ \text { DM28 } & 126 & 127 & 117 & 139 & 136 & 168 & 182 & 100 \\ \text { DM29 } & 83 & 78 & 84 & 87 & 92 & 111 & 127 & 73 \\ \text { DM30 } & 107 & 125 & 125 & 104 & 112 & 134 & 129 & 249 \\ \text { Mean } & 142 & 121 & 147 & 125 & 129 & 136 & 157 & 104 \\ \text { SD } & 188 & 66 & 77 & 58 & 53 & 44 & 67 & 35 \\ \text { LDH measurements } & \text { male beagles } & & & & & \\ & \text { LDH (IU/L) } & & & & & & & \\ \text { No. } & -6 \mathrm{D} & 0 \mathrm{D} & & & & & & \\ \text { DM01 } & 52 & \text { Pre } & 0.5 \mathrm{H} & 1 \mathrm{H} & 2 \mathrm{H} & 4 \mathrm{H} & 8 \mathrm{H} & 24 \mathrm{H} \\ \text { DM02 } & 57 & 54 & 47 & 34 & 67 & 45 & 53 & 99 \\ \text { DM03 } & 52 & 43 & 56 & 40 & 63 & 55 & 55 & 50 \\ \text { DM04 } & 47 & 71 & 70 & 64 & 161 & 93 & 89 & 75 \\ & & 130 & 66 & 52 & 93 & 76 & 75 & 196\end{array}$


Table 2 (continued)

\begin{tabular}{lllllllll}
\hline DM05 & 39 & 42 & 70 & 36 & 92 & 60 & 49 & 46 \\
DM06 & 61 & 47 & 66 & 50 & 55 & 74 & 51 & 72 \\
DM07 & 67 & 92 & 90 & 57 & 105 & 98 & 86 & 97 \\
DM08 & 51 & 44 & 57 & 42 & 53 & 47 & 85 & 94 \\
DM09 & 58 & 55 & 51 & 91 & 82 & 132 & 72 & 110 \\
DM10 & 71 & 53 & 62 & 66 & 62 & 93 & 55 & 139 \\
DM11 & 77 & 61 & 87 & 45 & 137 & 103 & 85 & 102 \\
DM12 & 127 & 56 & 58 & 120 & 39 & 81 & 85 & 38 \\
DM13 & 142 & 83 & 44 & 47 & 55 & 134 & 148 & 92 \\
DM14 & 46 & 43 & 72 & 40 & 37 & 43 & 47 & 55 \\
DM15 & 88 & 48 & 55 & 47 & 48 & 80 & 79 & 50 \\
DM16 & 62 & 40 & 68 & 59 & 49 & 40 & 84 & 31 \\
DM17 & 61 & 54 & 67 & 122 & 44 & 61 & 97 & 53 \\
DM18 & 45 & 32 & 72 & 90 & 61 & 73 & 55 & 45 \\
DM19 & 68 & 55 & 82 & 67 & 65 & 67 & 130 & 42 \\
DM20 & 54 & 42 & 56 & 90 & 52 & 71 & 80 & 47 \\
DM21 & 79 & 117 & 127 & 74 & 77 & 77 & 60 & 47 \\
DM22 & 30 & 69 & 74 & 66 & 42 & 50 & 43 & 49 \\
DM23 & 60 & 86 & 95 & 89 & 45 & 72 & 120 & 29 \\
DM24 & 100 & 62 & 65 & 93 & 30 & 52 & 34 & 31 \\
DM25 & 47 & 78 & 38 & 42 & 40 & 49 & 40 & 51 \\
DM26 & 57 & 63 & 64 & 41 & 46 & 67 & 61 & 42 \\
DM27 & 56 & 75 & 47 & 51 & 57 & 110 & 50 & 47 \\
DM28 & 64 & 93 & 39 & 76 & 56 & 60 & 42 & 46 \\
DM29 & 43 & 41 & 36 & 38 & 38 & 71 & 45 & 42 \\
DM30 & 52 & 105 & 87 & 38 & 36 & 56 & 42 & 56 \\
Mean & 64 & 64 & 66 & 62 & 63 & 73 & 70 & 66 \\
SD & 24 & 24 & 19 & 24 & 29 & 24 & 27 & 36 \\
\hline
\end{tabular}

cTnI measurements in female beagles

\begin{tabular}{|c|c|c|c|c|c|c|c|c|}
\hline & $\mathrm{cTnI}(\mathrm{ng} / \mathrm{mL})$ & & & & & & & \\
\hline & $-6 \mathrm{D}$ & $0 \mathrm{D}$ & & & & & & \\
\hline No. & - & Pre & $0.5 \mathrm{~h}$ & $1 \mathrm{~h}$ & $2 \mathrm{~h}$ & $4 \mathrm{~h}$ & $8 \mathrm{~h}$ & $24 \mathrm{~h}$ \\
\hline DF01 & 0.34 & 0.75 & 0.38 & 0.61 & 0.75 & 0.65 & 0.56 & 0.50 \\
\hline DF02 & - & 0.37 & 0.30 & 0.33 & 0.23 & 0.42 & - & 0.38 \\
\hline DF03 & - & - & - & - & 0.29 & - & 0.33 & - \\
\hline DF04 & - & - & - & 0.18 & - & - & - & - \\
\hline DF05 & - & - & - & - & - & 0.22 & - & - \\
\hline DF06 & 0.51 & 0.24 & 0.19 & 0.23 & 0.32 & 0.57 & 0.38 & 0.38 \\
\hline DF07 & - & - & 0.27 & - & - & - & 0.16 & - \\
\hline DF08 & - & - & - & 0.17 & - & - & 0.24 & 0.26 \\
\hline DF09 & - & - & - & 0.20 & - & - & 0.23 & - \\
\hline DF10 & 0.21 & - & 0.17 & - & - & - & - & - \\
\hline DF11 & - & 0.64 & 0.41 & 0.35 & 0.38 & 0.55 & 0.29 & 0.44 \\
\hline DF12 & 0.38 & 0.23 & 0.71 & 0.50 & 0.54 & 0.46 & 0.52 & 0.28 \\
\hline DF13 & 0.22 & 0.41 & - & 0.63 & 0.43 & 0.56 & 0.32 & 0.43 \\
\hline DF14 & 0.38 & 0.42 & 0.62 & 0.25 & 0.81 & 0.54 & 0.79 & 0.36 \\
\hline DF15 & - & 0.44 & 0.29 & 0.47 & - & 0.68 & 0.46 & 0.42 \\
\hline DF16 & 0.27 & - & 0.46 & 0.31 & 0.42 & - & 0.67 & 0.34 \\
\hline DF17 & - & 0.33 & - & 0.37 & - & 0.19 & - & 0.37 \\
\hline DF18 & 0.55 & 0.38 & 0.78 & 0.26 & 0.81 & 0.46 & 0.65 & - \\
\hline DF19 & 0.35 & 0.29 & 0.22 & 0.25 & - & 0.42 & - & - \\
\hline DF20 & 0.42 & 0.34 & 0.31 & 0.43 & 0.52 & 0.23 & 0.59 & - \\
\hline DF21 & - & 0.32 & - & 0.28 & - & - & - & - \\
\hline DF22 & - & - & 0.42 & - & 0.29 & 0.20 & 0.22 & - \\
\hline DF23 & 0.32 & 0.40 & 0.33 & 0.65 & 0.50 & 0.82 & 0.39 & 0.47 \\
\hline DF24 & - & - & - & - & 0.22 & - & - & - \\
\hline DF25 & - & 0.16 & - & - & - & 0.22 & 0.18 & 0.21 \\
\hline DF26 & - & - & - & - & - & - & 0.32 & - \\
\hline DF27 & - & - & - & 0.21 & - & - & - & - \\
\hline DF28 & 0.97 & 0.79 & 0.88 & 0.92 & 0.66 & 0.84 & 1.00 & 0.66 \\
\hline DF29 & - & 0.23 & - & - & - & 0.25 & - & - \\
\hline DF30 & 0.31 & 0.19 & 0.38 & 0.21 & 0.25 & - & 0.22 & - \\
\hline Mean & 0.17 & 0.23 & 0.24 & 0.26 & 0.25 & 0.28 & 0.28 & 0.18 \\
\hline SD & 0.24 & 0.23 & 0.26 & 0.23 & 0.27 & 0.28 & 0.27 & 0.21 \\
\hline
\end{tabular}

Table 2 (continued)

cTnT measurements in female beagles

$$
\mathrm{cTnT}(\mathrm{ng} / \mathrm{L})
$$

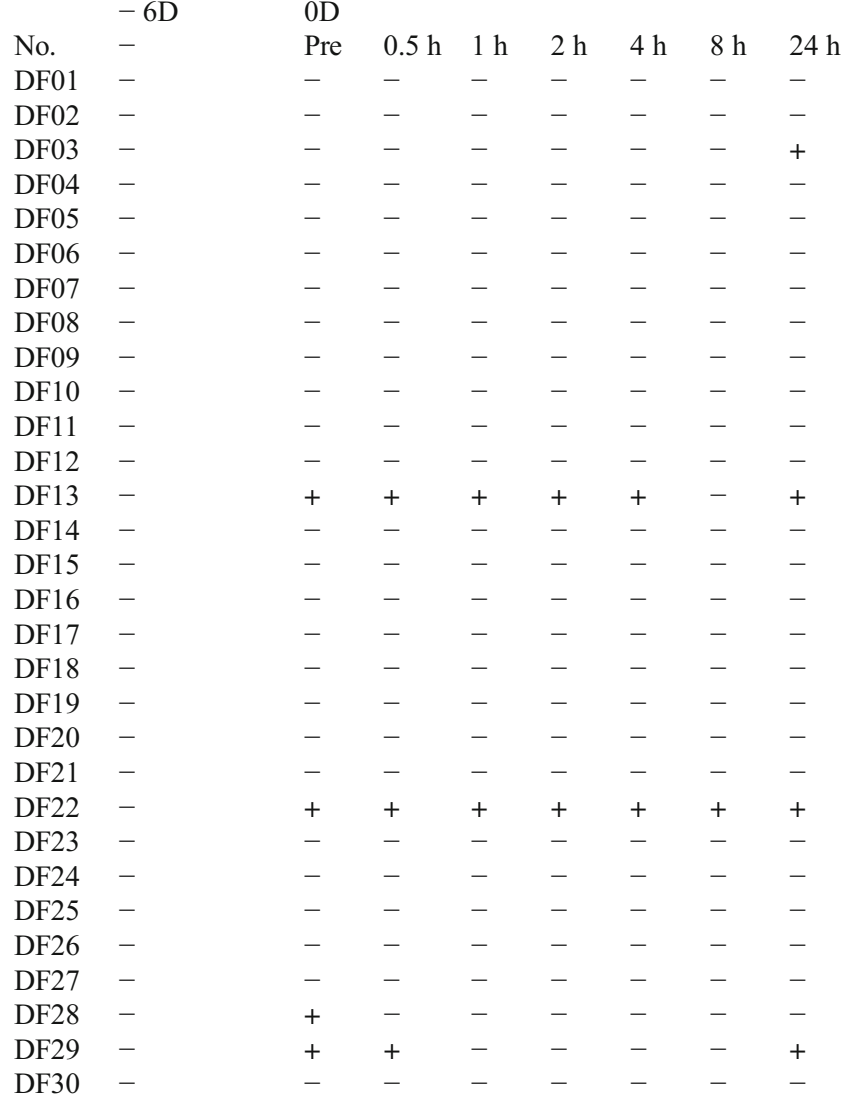

CK (IU/L) $-6 \mathrm{D}$

No.

DF01 159

DF02 131

DF03 104

DF04 108

DF05 96

DF06 96

DF07 122

DF08 103

DF09 111

DF10 90

DF11 152

DF12 119

DF13 68

DF14 122

DF15 112

DF16 177

DF17 132

DF18 141

DF19 114

DF20 291

DF21 83

DF22 85

DF23 89

DF24 122

DF25 148

DF26 82

DF27 86

$\begin{array}{lllllll}\text { OD } & & & & & & \\ \text { Pre } & 0.5 \mathrm{~h} & 1 \mathrm{~h} & 2 \mathrm{~h} & 4 \mathrm{~h} & 8 \mathrm{~h} & 24 \mathrm{~h} \\ 130 & 226 & 136 & 142 & 157 & 205 & 144 \\ 129 & 140 & 140 & 136 & 156 & 251 & 125 \\ 83 & 92 & 86 & 96 & 96 & 97 & 89 \\ 101 & 93 & 87 & 86 & 96 & 117 & 93 \\ 78 & 69 & 74 & 89 & 84 & 118 & 83 \\ 145 & 131 & 102 & 99 & 138 & 118 & 120 \\ 129 & 105 & 106 & 105 & 111 & 113 & 130 \\ 148 & 121 & 105 & 93 & 112 & 109 & 153 \\ 84 & 81 & 78 & 82 & 93 & 112 & 101 \\ 89 & 90 & 151 & 95 & 110 & 104 & 98 \\ 160 & 164 & 157 & 158 & 148 & 136 & 155 \\ 122 & 123 & 106 & 102 & 108 & 106 & 116 \\ 72 & 66 & 62 & 72 & 63 & 65 & 86 \\ 112 & 127 & 97 & 113 & 129 & 150 & 115 \\ 137 & 119 & 115 & 122 & 143 & 346 & 160 \\ 151 & 243 & 145 & 155 & 153 & 316 & 130 \\ 141 & 143 & 139 & 129 & 177 & 157 & 113 \\ 128 & 125 & 125 & 143 & 126 & 144 & 124 \\ 127 & 96 & 94 & 94 & 105 & 146 & 119 \\ 153 & 99 & 102 & 108 & 119 & 100 & 88 \\ 87 & 89 & 87 & 113 & 79 & 79 & 82 \\ 113 & 80 & 83 & 110 & 98 & 84 & 79 \\ 79 & 86 & 78 & 88 & 103 & 112 & 80 \\ 129 & 146 & 121 & 123 & 133 & 132 & 115 \\ 98 & 115 & 90 & 119 & 117 & 85 & 109 \\ 83 & 77 & 68 & 86 & 91 & 110 & 76 \\ 86 & 87 & 91 & 84 & 101 & 95 & 85\end{array}$


Table 2 (continued)

\begin{tabular}{|c|c|c|c|c|c|c|c|c|}
\hline DF28 & 263 & 92 & 127 & 78 & 80 & 91 & 81 & 327 \\
\hline DF29 & 117 & 165 & 139 & 136 & 135 & 128 & 139 & 103 \\
\hline DF30 & 103 & 92 & 149 & 213 & 123 & 245 & 108 & 87 \\
\hline Mean & 124 & 115 & 118 & 108 & 109 & 120 & 135 & 116 \\
\hline SD & 48 & 28 & 40 & 32 & 23 & 35 & 64 & 46 \\
\hline \multicolumn{9}{|c|}{$\begin{array}{l}\text { LDH measurements in female beagles } \\
\text { LDH (IU/L) }\end{array}$} \\
\hline & $-6 \mathrm{D}$ & 0D & & & & & & \\
\hline No. & - & Pre & $0.5 \mathrm{~h}$ & $1 \mathrm{~h}$ & $2 \mathrm{~h}$ & $4 \mathrm{~h}$ & $8 \mathrm{~h}$ & $24 \mathrm{~h}$ \\
\hline DF01 & 66 & 45 & 67 & 42 & 44 & 53 & 80 & 61 \\
\hline DF02 & 47 & 44 & 43 & 45 & 37 & 59 & 59 & 56 \\
\hline DF03 & 60 & 55 & 61 & 55 & 56 & 90 & 62 & 65 \\
\hline DF04 & 49 & 51 & 57 & 46 & 42 & 60 & 82 & 67 \\
\hline DF05 & 42 & 57 & 36 & 50 & 73 & 71 & 102 & 45 \\
\hline DF06 & 45 & 47 & 48 & 43 & 41 & 57 & 47 & 54 \\
\hline DF07 & 60 & 107 & 44 & 38 & 36 & 62 & 56 & 69 \\
\hline DF08 & 47 & 145 & 38 & 40 & 35 & 59 & 54 & 75 \\
\hline DF09 & 58 & 53 & 52 & 44 & 46 & 55 & 76 & 55 \\
\hline DF10 & 52 & 57 & 43 & 57 & 48 & 95 & 78 & 51 \\
\hline DF11 & 76 & 68 & 65 & 64 & 81 & 56 & 60 & 79 \\
\hline DF12 & 49 & 43 & 49 & 43 & 43 & 77 & 54 & 59 \\
\hline DF13 & 42 & 47 & 52 & 37 & 66 & 42 & 46 & 96 \\
\hline DF14 & 47 & 58 & 84 & 34 & 58 & 78 & 58 & 81 \\
\hline DF15 & 66 & 108 & 43 & 38 & 57 & 97 & 97 & 88 \\
\hline DF16 & 94 & 61 & 58 & 49 & 69 & 65 & 100 & 148 \\
\hline DF17 & 70 & 64 & 50 & 54 & 45 & 150 & 85 & 64 \\
\hline DF18 & 91 & 81 & 65 & 71 & 129 & 76 & 87 & 82 \\
\hline DF19 & 93 & 72 & 50 & 47 & 47 & 62 & 121 & 132 \\
\hline DF20 & 110 & 66 & 48 & 45 & 65 & 93 & 90 & 69 \\
\hline DF21 & 47 & 53 & 57 & 60 & 154 & 57 & 54 & 69 \\
\hline DF22 & 41 & 125 & 39 & 43 & 82 & 68 & 37 & 44 \\
\hline DF23 & 49 & 43 & 72 & 48 & 43 & 63 & 44 & 45 \\
\hline DF24 & 54 & 87 & 113 & 57 & 50 & 72 & 49 & 71 \\
\hline DF25 & 54 & 47 & 100 & 43 & 123 & 122 & 34 & 83 \\
\hline DF26 & 39 & 53 & 42 & 33 & 60 & 52 & 44 & 42 \\
\hline DF27 & 51 & 51 & 43 & 63 & 38 & 76 & 42 & 45 \\
\hline DF28 & 75 & 81 & 53 & 46 & 41 & 56 & 33 & 61 \\
\hline DF29 & 63 & 162 & 66 & 58 & 51 & 54 & 50 & 69 \\
\hline DF30 & 41 & 40 & 127 & 95 & 52 & 70 & 41 & 46 \\
\hline Mean & 59 & 69 & 59 & 50 & 60 & 72 & 64 & 69 \\
\hline SD & 18 & 31 & 21 & 12 & 28 & 22 & 23 & 24 \\
\hline
\end{tabular}

cTnI: Values below the lower limit of quantification (BLOQ) $(0.156 \mathrm{ng} / \mathrm{mL})$ were shown as "-" and regarded as $0 \mathrm{ng} / \mathrm{mL}$ in calculation

cTnT: Values below the lower limit of quantification (BLOQ) (50 ng/L) were shown as "-". Values between 50 and $100 \mathrm{ng} / \mathrm{L}$ were shown as "+"

\section{Cynomolgus monkey}

One female (CF01) showed a higher level of serum cTnI at all points (Table 3 ). Three males (CM02, CM04, and CM08) and one female (CF09) showed sporadically low levels of cTnI through the examination period (Table 3 ). Only two males (CM07 and CM08) showed low but detectable blood cTnT values (Table 3). Although the higher levels of CK or LDH were detected sporadically, no correspondences were noted in the changes in $\mathrm{cTnI}$ or $\mathrm{cTnT}$ levels (Table 3 ).
Table 3 Measurements in cynomolgus monkeys

cTnI measurements in male cynomolgus monkeys

$\mathrm{cTnI}(\mathrm{ng} / \mathrm{mL})$

$-13 \mathrm{D} 0 \mathrm{D}$

$\begin{array}{lllllllll}\text { No. } & - & \text { Pre } & 0.5 \mathrm{~h} & 1 \mathrm{~h} & 2 \mathrm{~h} & 4 \mathrm{~h} & 8 \mathrm{~h} & 24 \mathrm{~h} \\ \mathrm{CM} 1 & - & - & - & - & - & - & - & - \\ \mathrm{CM} 2 & - & - & - & - & - & - & 0.20 & - \\ \mathrm{CM} 3 & - & - & - & - & - & - & - & - \\ \mathrm{CM} 4 & - & 0.16 & 0.26 & 0.20 & - & - & 0.17 & 0.22 \\ \mathrm{CM} 05 & - & - & - & - & - & - & - & - \\ \mathrm{CM} 6 & - & - & - & - & - & - & - & - \\ \mathrm{CM} 07 & - & - & - & - & - & - & - & - \\ \mathrm{CM} 08 & - & - & - & - & - & 0.17 & - & 0.16 \\ \mathrm{CM} 9 & - & - & - & - & - & - & - & - \\ \mathrm{CM} 10 & - & - & - & - & - & - & - & - \\ \text { Mean } & 0.00 & 0.02 & 0.03 & 0.02 & 0.00 & 0.02 & 0.04 & 0.04 \\ \text { SD } & 0.00 & 0.05 & 0.08 & 0.06 & 0.00 & 0.05 & 0.07 & 0.08\end{array}$

$\mathrm{cTnT}$ measurements in male cynomolgus monkeys cTnT (ng/L)

$-13 \mathrm{D} 0 \mathrm{D}$

No. - Pre $0.5 \mathrm{~h} \quad 1 \mathrm{~h} \quad 2 \mathrm{~h} \quad 4 \mathrm{~h} \quad 8 \mathrm{~h} \quad 24 \mathrm{~h}$

$\begin{array}{lllllllllllll}\mathrm{CM} 01 & - & - & - & - & - & - & - & - & - & -\end{array}$

$\begin{array}{lllllllllllll}\mathrm{CM} 02 & - & - & - & - & - & - & - & - & -\end{array}$

$\begin{array}{lllllllllllll}\mathrm{CM} 03 & - & - & - & - & - & - & - & - & - & -\end{array}$

$\begin{array}{llllllllllll}\mathrm{CM} 04 & - & - & - & - & - & - & - & - & -\end{array}$

$\begin{array}{lllllllllll}\mathrm{CM} 05 & - & - & - & - & - & - & - & -\end{array}$

$\begin{array}{llllllllllll}\mathrm{CM} 06 & - & - & - & - & - & - & - & - & -\end{array}$

$\mathrm{CM} 07+\quad+\quad+\quad+\quad+\quad+\quad+$

$\begin{array}{lllllllllllll}\mathrm{CM} 08 & + & - & - & - & + & - & - & -\end{array}$

CM09

CM10 -

CK measurements in male cynomolgus monkeys

$$
\text { CK (IU/L) }
$$$$
-13 \mathrm{D} \quad 0 \mathrm{D}
$$

$\begin{array}{lllllllll}\text { No. } & - & \text { Pre } & 0.5 \mathrm{~h} & 1 \mathrm{~h} & 2 \mathrm{~h} & 4 \mathrm{~h} & 8 \mathrm{~h} & 24 \mathrm{~h} \\ \text { CM01 } & 970 & 328 & 581 & 1283 & 2596 & 5238 & 4153 & 795 \\ \text { CM02 } & 141 & 263 & 707 & 1144 & 1817 & 3639 & 8706 & 807 \\ \text { CM03 } & 188 & 216 & 553 & 824 & 951 & 1823 & 1779 & 1004 \\ \text { CM04 } & 399 & 421 & 364 & 512 & 373 & 868 & 959 & 333 \\ \text { CM05 } & 629 & 174 & 1261 & 715 & 612 & 1342 & 845 & 310 \\ \text { CM06 } & 140 & 366 & 545 & 1460 & 268 & 634 & 2620 & 424 \\ \text { CM07 } & 356 & 302 & 928 & 1723 & 2589 & 4341 & 6119 & 1831 \\ \text { CM08 } & 216 & 283 & 385 & 566 & 618 & 1161 & 2495 & 870 \\ \text { CM09 } & 458 & 379 & 554 & 1289 & 1111 & 1232 & 2785 & 577 \\ \text { CM10 } & 179 & 164 & 221 & 524 & 251 & 699 & 520 & 507 \\ \text { Mean } & 368 & 290 & 610 & 1004 & 1119 & 2098 & 3098 & 746 \\ \text { SD } & 252 & 82 & 284 & 410 & 860 & 1586 & 2458 & 426\end{array}$

LDH measurements in male cynomolgus monkeys

LDH (IU/L)

$-13 \mathrm{D} \quad 0 \mathrm{D}$

No. $\quad-\quad$ Pre $\quad 0.5 \mathrm{~h} \quad 1 \mathrm{~h} \quad 2 \mathrm{~h} \quad 4 \mathrm{~h} \quad 8 \mathrm{~h} \quad 24 \mathrm{~h}$ 
Table 3 (continued)

\begin{tabular}{lllllllll}
\hline CM01 & 513 & 457 & 633 & 847 & 707 & 999 & 1045 & 790 \\
CM02 & 241 & 447 & 497 & 640 & 740 & 904 & 1207 & 532 \\
CM03 & 365 & 495 & 623 & 656 & 899 & 782 & 717 & 579 \\
CM04 & 618 & 455 & 349 & 733 & 298 & 377 & 341 & 253 \\
CM05 & 241 & 199 & 298 & 454 & 291 & 441 & 331 & 260 \\
CM06 & 281 & 355 & 684 & 711 & 407 & 469 & 833 & 327 \\
CM07 & 298 & 303 & 465 & 581 & 687 & 892 & 990 & 537 \\
CM08 & 262 & 293 & 403 & 374 & 360 & 513 & 619 & 367 \\
CM09 & 354 & 343 & 419 & 637 & 508 & 545 & 679 & 372 \\
CM10 & 236 & 244 & 273 & 544 & 288 & 524 & 345 & 342 \\
Mean & 341 & 359 & 464 & 618 & 519 & 645 & 711 & 436 \\
SD & 122 & 96 & 137 & 130 & 212 & 214 & 296 & 161
\end{tabular}

cTnI measurements in female cynomolgus monkeys cTnI (ng/mL)

\begin{tabular}{|c|c|c|c|c|c|c|c|c|}
\hline & $-13 \mathrm{D}$ & $0 \mathrm{D}$ & & & & & & \\
\hline No. & - & Pre & $0.5 \mathrm{~h}$ & $1 \mathrm{~h}$ & $2 \mathrm{~h}$ & $4 \mathrm{~h}$ & $8 \mathrm{~h}$ & $24 \mathrm{~h}$ \\
\hline CF01 & 0.72 & 0.95 & 0.88 & 0.77 & 0.84 & 0.90 & 0.83 & 0.82 \\
\hline CF02 & - & - & - & - & - & - & - & - \\
\hline CF03 & - & - & - & - & - & - & - & - \\
\hline CF04 & - & - & - & - & - & - & - & - \\
\hline CF05 & - & - & - & - & - & - & - & - \\
\hline CF06 & - & - & - & - & - & - & - & - \\
\hline CF07 & - & - & - & - & - & - & - & - \\
\hline CF08 & - & - & - & - & - & - & - & - \\
\hline CF09 & - & 0.19 & - & - & - & - & - & - \\
\hline CF10 & - & - & - & - & - & - & - & - \\
\hline Mean & 0.07 & 0.11 & 0.09 & 0.08 & 0.08 & 0.09 & 0.08 & 0.08 \\
\hline SD & 0.22 & 0.28 & 0.26 & 0.23 & 0.25 & 0.27 & 0.25 & 0.25 \\
\hline
\end{tabular}

cTnT measurements in female cynomolgus monkeys cTnT (ng/L)

$-13 \mathrm{D} \quad 0 \mathrm{D}$

$\begin{array}{lllllllll}\text { No. } & - & \text { Pre } & 0.5 \mathrm{~h} & 1 \mathrm{~h} & 2 \mathrm{~h} & 4 \mathrm{~h} & 8 \mathrm{~h} & 24 \mathrm{~h} \\ \text { CF01 } & - & - & - & - & - & - & - & - \\ \mathrm{CF02} & - & - & - & - & - & - & - & - \\ \mathrm{CF03} & - & - & - & - & - & - & - & - \\ \text { CF04 } & - & - & - & - & - & - & - & - \\ \text { CF05 } & - & - & - & - & - & - & - & - \\ \text { CF06 } & - & - & - & - & - & - & - & - \\ \text { CF07 } & - & - & - & - & - & - & - & - \\ \text { CF08 } & - & - & - & - & - & - & - & - \\ \text { CF09 } & - & - & - & - & - & - & - & - \\ \text { CF10 } & - & - & - & - & - & - & - & -\end{array}$

CK measurements in female cynomolgus monkeys

CK (IU/L)

$\begin{array}{lllllllll} & -13 \mathrm{D} & \text { 0D } & & & & & & \\ \text { No. } & - & \text { Pre } & 0.5 \mathrm{~h} & 1 \mathrm{~h} & 2 \mathrm{~h} & 4 \mathrm{~h} & 8 \mathrm{~h} & 24 \mathrm{~h} \\ \text { CF01 } & 92 & 220 & 160 & 271 & 943 & 2860 & 1301 & 286 \\ \text { CF02 } & 125 & 113 & 162 & 691 & 341 & 497 & 837 & 303 \\ \text { CF03 } & 166 & 231 & 225 & 320 & 298 & 545 & 700 & 249 \\ \text { CF04 } & 425 & 1406 & 2510 & 2941 & 4094 & 7187 & 4558 & 1430 \\ \text { CF05 } & 141 & 213 & 218 & 253 & 293 & 336 & 294 & 257 \\ \text { CF06 } & 317 & 393 & 360 & 569 & 507 & 565 & 964 & 260 \\ \text { CF07 } & 638 & 391 & 1239 & 1532 & 1903 & 1698 & 2142 & 550 \\ \text { CF08 } & 290 & 405 & 230 & 439 & 508 & 1196 & 994 & 381 \\ \text { CF09 } & 255 & 1512 & 2180 & 4080 & 3935 & 7108 & 8755 & 2369 \\ \text { CF10 } & 190 & 449 & 1442 & 546 & 572 & 503 & 727 & 316 \\ \text { Mean } & 264 & 533 & 873 & 1164 & 1339 & 2250 & 2127 & 640 \\ \text { SD } & 158 & 475 & 859 & 1250 & 1412 & 2555 & 2494 & 669\end{array}$

LDH measurements in female cynomolgus monkeys

LDH (IU/L)

$$
-13 \mathrm{D} \text { 0D }
$$

$\begin{array}{lllllllll}\text { No. } & - & \text { Pre } & 0.5 \mathrm{~h} & 1 \mathrm{~h} & 2 \mathrm{~h} & 4 \mathrm{~h} & 8 \mathrm{~h} & 24 \mathrm{~h} \\ \text { CF01 } & 197 & 239 & 263 & 363 & 391 & 795 & 582 & 265\end{array}$

$\begin{array}{lllllllll}\mathrm{CF} 02 & 386 & 258 & 395 & 444 & 440 & 366 & 345 & 326\end{array}$

Table 3 (continued)

\begin{tabular}{lllllllll}
\hline CF03 & 553 & 295 & 387 & 420 & 476 & 421 & 405 & 461 \\
CF04 & 454 & 636 & 792 & 876 & 962 & 1462 & 1053 & 816 \\
CF05 & 231 & 247 & 258 & 268 & 268 & 299 & 271 & 253 \\
CF06 & 326 & 288 & 313 & 444 & 352 & 378 & 406 & 306 \\
CF07 & 331 & 359 & 547 & 627 & 670 & 595 & 564 & 377 \\
CF08 & 306 & 286 & 285 & 312 & 340 & 448 & 406 & 351 \\
CF09 & 282 & 468 & 563 & 797 & 772 & 1011 & 1038 & 623 \\
CF10 & 186 & 203 & 298 & 251 & 272 & 289 & 282 & 232 \\
Mean & 325 & 328 & 410 & 480 & 494 & 606 & 535 & 401 \\
SD & 109 & 124 & 165 & 206 & 220 & 360 & 273 & 177 \\
\hline
\end{tabular}

cTnI: Values below the lower limit of quantification (BLOQ) $(0.156 \mathrm{ng} / \mathrm{mL})$ were shown as "-_" and regarded as $0 \mathrm{ng} / \mathrm{mL}$ in calculation

cTnT: Values below the lower limit of quantification (BLOQ) (50 ng/L) were shown as "-". Values between 50 and $100 \mathrm{ng} / \mathrm{L}$ were shown as "+"

\section{Discussion}

In this study, we revealed the extent of individual differences in baseline levels and operational effects in Sprague Dawley rats, beagle dogs, and cynomolgus monkeys from repeated blood samplings using needle cylinders under restraint up until $24 \mathrm{~h}$ after a single oral dose of $0.5 \mathrm{w} / \mathrm{v} \%$ methyl cellulose solution as a vehicle. For the rats, although some animals showed temporal elevations 2-4 h after dosing, cTnI levels were BLOQ at almost all examination points. In contrast, there were substantially larger individual differences in baseline levels of $\mathrm{cTnI}$ in dogs (greater than 20-fold) and cynomolgus monkeys (greater than 5-fold). cTnI values fluctuated around individual baselines without clear correlations in timing or with CK and LDH elevations seen in some animals. This suggests that these fluctuations of cTnI values were not caused by the experimental procedures, neither treatment nor operational, and thus individual variations in baseline levels need to be taken into account when evaluating cTnI levels in blood collected. Based on these results, we propose the criteria shown here:

For rats, we can evaluate cTnI levels from blood sampling $24 \mathrm{~h}$ after treatment by simply adopting the BLOQ as a criterion for treatment-related effects (e.g., compoundinduced effects after drug administration), without needing to consider individual variations or operational effects. When we evaluate cTnI levels from blood sampling collected periodically within the same day of treatment, however, we need to reject temporal elevations as operational effects, based on historical background data defined at each facility (e.g., $0.02 \mathrm{ng} / \mathrm{mL}$, if based on this study).

For dogs and cynomolgus monkeys, we can adopt the following criteria.

1. For all animals in a study, calculate the individual maximum untreated level (IULmax), the individual minimum untreated level (IULmin), and individual untreated range (IULrange; IULmax - IULmin), based on measurements 
at all-time points for control animals and at time points before treatment for treated animals.

2. Define the criterion of level $(\mathrm{CoL})$ as the highest IULmax and the criterion of variation $(\mathrm{CoV})$ as the highest IULrange in the study.

3. A measured value (MV) taken during the treatment period is considered to have resulted from treatment if MV > $\mathrm{CoL}$ and $(\mathrm{MV}-\mathrm{IULmin})>\mathrm{CoV}$.

For example, considering Table 3 as data of control animals, $\mathrm{CoL}$ and $\mathrm{CoV}$ are defined as $0.26 \mathrm{ng} / \mathrm{mL}$ (IULmax of $\mathrm{CM} 04$ at $0.5 \mathrm{~h}$ ) and $0.10 \mathrm{ng} / \mathrm{mL}$ (the highest IULrange; 0.26$0.16 \mathrm{ng} / \mathrm{mL}$ of CM04), respectively. We regarded BLOQ values as $0.16 \mathrm{ng} / \mathrm{mL}$ (based on the LOQ value) in this calculation, to avoid overestimating IULrange. Now, suppose that one animal showed a MV of $0.50 \mathrm{ng} / \mathrm{mL}$ at some point after treatment and its IULmin was $0.16 \mathrm{ng} / \mathrm{mL}$. In this case, the MV is considered to have resulted from treatment, since MV $(0.50 \mathrm{ng} / \mathrm{mL})>\mathrm{CoL}(0.26 \mathrm{ng} / \mathrm{mL})$ and $\mathrm{MV}$ $(0.50 \mathrm{ng} / \mathrm{mL})-\operatorname{IULmin}(0.16 \mathrm{ng} / \mathrm{mL})>\operatorname{CoV}(0.10 \mathrm{ng} / \mathrm{mL})$.

These criteria could minimize false positives. However, they may not be applied in cases where a small number of animals show considerably higher baseline levels than the others, since inclusion of such animals would lead to underestimation of the treatment-related changes. In such cases, excluding outliers prior to the start of a study could minimize individual variations in baseline levels.

Regarding cTnT, the values were mostly BLOQ, more frequently than those of cTnI. This might be attributable to differences in the measurement systems used. For rats, all cTnT measurements were BLOQ, and therefore, we do not need to consider individual variation or operational effects. For dogs and cynomolgus monkeys, however, we should use the same approach with cTnI, since some animals showed levels exceeding LOQ.

In conclusion, we proposed criteria to distinguish treatmentrelated effects from individual differences and operational effects in Sprague-Dawley rats, beagle dogs, and cynomolgus monkeys. We admit that our study lacks data from animals after treatment of myocardial infarction-inducing compounds. In the future, such positive control data would be needed and would help us establish more accurate criteria.

Funding This study was not funded by any third parties.

\section{Compliance with ethical standards}

Conflict of interest The authors declare that they have no conflict of interest.

Ethical approval The animal experiments within this study were approved by the Institutional Animal Care and Use Committee of Shin Nippon Biomedical Laboratories and/or Astellas Pharma Inc., and were performed in accordance with the animal welfare guidelines thereof.
Procedures specific to each animal species are described separately in the Material and Methods subsection.

Open Access This article is distributed under the terms of the Creative Commons Attribution 4.0 International License (http:// creativecommons.org/licenses/by/4.0/), which permits unrestricted use, distribution, and reproduction in any medium, provided you give appropriate credit to the original author(s) and the source, provide a link to the Creative Commons license, and indicate if changes were made.

\section{References}

Berridge BR et al (2009) A translational approach to detecting druginduced cardiac injury with cardiac troponins: Consensus and recommendations from the Cardiac Troponins Biomarker Working Group of the Health and Environmental Sciences Institute. Am Heart J 158:21-29. https://doi.org/10.1016/j.ahj.2009.04.020

Hausner EA, Hicks KA, Leighton JK, Szarfman A, Thompson AM, Harlow P (2013) Qualification of cardiac troponins for nonclinical use: a regulatory perspective. Regul Toxicol Pharmacol 67:108-114. https://doi.org/10.1016/j.yrtph.2013.07.006

Herman EH et al (2001) The use of serum levels of cardiac troponin T to compare the protective activity of dexrazoxane against doxorubicinand mitoxantrone-induced cardiotoxicity. Cancer Chemother Pharmacol 48:297-304

Mahajan VS, Jarolim P (2011) How to interpret elevated cardiac troponin levels. Circulation 124:2350-2354. https://doi.org/10.1161/ CIRCULATIONAHA.111.023697

Newby LK et al (2011) Troponin measurements during drug development - considerations for monitoring and management of potential cardiotoxicity: an educational collaboration among the cardiac safety research consortium, the Duke Clinical Research Institute, and the US Food and Drug Administration. Am Heart J 162:64-73. https:// doi.org/10.1016/j.ahj.2011.04.005

O'Brien PJ et al (2006) Cardiac troponin I is a sensitive, specific biomarker of cardiac injury in laboratory animals. Lab Anim 40: 153-171. https://doi.org/10.1258/002367706776319042

Pierson JB et al (2013) A public-private consortium advances cardiac safety evaluation: achievements of the HESI Cardiac Safety Technical Committee. J Pharmacol Toxicol Methods 68:7-12. https://doi.org/10.1016/j.vascn.2013.03.008

Schultze AE et al (2009) Longitudinal studies of cardiac troponin-I concentrations in serum from male Sprague Dawley rats: baseline reference ranges and effects of handling and placebo dosing on biological variability. Toxicol Pathol 37:754-760. https://doi.org/ $10.1177 / 0192623309343777$

Schultze AE et al (2015) Longitudinal studies of cardiac troponin I concentrations in serum from male cynomolgus monkeys: resting values and effects of oral and intravenous dosing on biologic variability. Vet Clin Pathol/Am Soc Vet Clin Pathol 44:465-471. https:// doi.org/10.1111/vcp. 12272

Undhad VV, Fefar DT, Jivani BM, Gupta H, Ghodasara DJ, Joshi BP, Prajapati KS (2012) Cardiac troponin: an emerging cardiac biomarker in animal health. Vet World 5:508-511. https://doi.org/10.5455/ vetworld.2012.508-511 